\title{
Effect of Exercise on Gait Kinematics and Kinetics in Patients with Chronic Ischaemic Stroke
}

\section{Styliani Fotiadou1, Ioannis Kouroumichakis², Thomas Besios ${ }^{3}$, Nikolaos Papanas ${ }^{4}$, Erasmia Giannakou' ${ }^{1}$, Vassilios Gourgoulis ${ }^{1}$, Nikolaos Aggeloussis ${ }^{1}$}

\author{
${ }^{1}$ Department of Physical Education and Sport Science, Democritus University of Thrace, Komotini, Greece \\ ${ }^{2}$ Private Internal Medicine Office, Alexandroupolis, Greece \\ ${ }^{3}$ Department of Physical Therapy, University of Thessaly, Lamia, Greece \\ ${ }^{4}$ Second Department of Internal Medicine, Democritus University of Thrace, Alexandroupolis, Greece \\ Email: s_fotiadou@yahoo.gr
}

How to cite this paper: Fotiadou, S., Kouroumichakis, I., Besios, T., Papanas, N., Giannakou, E., Gourgoulis, V. and Aggeloussis, N. (2019) Effect of Exercise on Gait Kinematics and Kinetics in Patients with Chronic Ischaemic Stroke. Open Journal of Therapy and Rehabilitation, 7, 140-150. https://doi.org/10.4236/ojtr.2019.74010

Received: September 23, 2019

Accepted: November 1, 2019

Published: November 4, 2019

Copyright $\odot 2019$ by author(s) and Scientific Research Publishing Inc. This work is licensed under the Creative Commons Attribution International License (CC BY 4.0).

http://creativecommons.org/licenses/by/4.0/

\begin{abstract}
Introduction: In 2014, American Heart Association and American Stroke Association (AHA/ASA) issued exercise guidelines for stroke patients. Aim of the Study: To study the effects of an exercise programme based on AHA/ ASA guidelines, on gait kinematics and kinetics in patients with chronic ischemic stroke. Materials and Methods: Twelve stroke patients, $67.33 \pm$ 9.14 years old, followed an 8-week exercise programme, with 3 hourly sessions per week, consisting of strength, endurance and flexibility training, as well as neuromuscular activities. Patients' gait kinematics and kinetics were evaluated before and after the intervention using a 3-dimensional gait analysis system. Results: In most cases, patients in the intervention group showed significant increase or no change in gait kinematics, significant increase in joint moments at the anterior-posterior plane during support phase, and non-significant change in the frontal and transverse planes kinetics. Conclusions: Exercise prevented further deterioration and/or led to improved walking pattern.
\end{abstract}

\section{Keywords}

Stroke, Exercise, Rehabilitation, Gait Analysis

\section{Introduction}

It is estimated that one in 5 women and one in 6 men will sustain a stroke up to the age of 75 years [1]. The main purpose of rehabilitation in such patients is to achieve the maximum possible personal performance, physical and psychologi- 
cal, with the ultimate goal of regaining a level of functional independence that will allow them to be re-integrated into social life as much as possible [2]. However, stroke patients often adopt a sedentary lifestyle [3] [4] [5] [6]. This may be attributed to 1) factors associated with patients themselves, such as depression, lack of interest or motivation, decreased perception, decreased confidence, ignorance that exercise is possible and desirability and fear of falls, of a new stroke or other undesirable effects; 2) practical factors, such as lack of support from family or other social actors, inability to access exercise sites, inadequate public transport, health professionals' ignorance of the availability of physical activity services; 3) financial cost [7] [8] [9] [10] [11]. Conversely, exercise in groups may improve patient motivation [12].

In 2014, the council of the American Heart Association and the American Stroke association (AHA/ASA) revised the exercise recommendations for stroke patients at all stages of their recovery [13]. Therefore, the aim of this study was to assess the effect of an exercise programme based on these recommendations on gait kinematics and kinetics of ischaemic stroke patients in the chronic phase of recovery.

\section{Patients and Methods}

\subsection{Sample}

Twelve patients, seven men and five women, aged $64.42 \pm 8.59$ years, who were hospitalised due to stroke in a General Hospital participated in the study. Nine of them had mild right paresis and three left paresis. Patients' characteristics are presented in Table 1. The study was approved by the institutional ethics committee and patient gave their informed consent.

Table 1. Patient characteristics.

\begin{tabular}{cccccccccc}
\hline Group & Gender & $\begin{array}{c}\text { Age } \\
(\text { years })\end{array}$ & $\begin{array}{c}\text { Height } \\
(\mathrm{cm})\end{array}$ & $\begin{array}{c}\text { Weight } \\
(\mathrm{kgr})\end{array}$ & $\begin{array}{c}\text { Paretic } \\
\text { side }\end{array}$ & $\begin{array}{c}\text { Time since } \\
\text { stroke (months) }\end{array}$ & mRS & BI & NIHSS \\
\hline C & M & 69 & 169.5 & 87 & $\mathrm{R}$ & 17 & 2 & 95 & 3 \\
I & F & 58 & 141 & 60 & $\mathrm{R}$ & 12 & 3 & 80 & 4 \\
C & M & 73 & 162 & 66 & $\mathrm{R}$ & 13 & 3 & 80 & 4 \\
I & M & 67 & 160 & 69 & $\mathrm{R}$ & 18 & 2 & 100 & 3 \\
C & M & 76 & 174 & 99 & $\mathrm{~L}$ & 14 & 2 & 100 & 2 \\
C & F & 52 & 152 & 63 & $\mathrm{R}$ & 12 & 2 & 90 & 4 \\
I & F & 53 & 173 & 105 & $\mathrm{R}$ & 15 & 1 & 100 & 2 \\
C & M & 61 & 169 & 80 & L & 14 & 1 & 100 & 2 \\
I & M & 63 & 162 & 71 & R & 17 & 2 & 85 & 4 \\
I & F & 73 & 152 & 63 & L & 14 & 2 & 100 & 2 \\
C & M & 73 & 158.5 & 77 & R & 13 & 3 & 80 & 4 \\
I & F & 55 & 153 & 82 & R & 14 & 2 & 100 & 2 \\
\hline
\end{tabular}

C: Control group, I: Intervention group, M: Male, F: Female, R: Right, L: Left, NIHSS: National Institutes of Health Scale, mRS: modified Rankin Scale, BI: Barthel Index. 
Inclusion criteria were: 1) hospitalisation for ischaemic stroke 12 to 18 months before initial assessment, 2) ability to walk continuously at least $20 \mathrm{~m}$ without aids or orthotics, 3) ability to understand and follow simple instructions, 4) adequate vision and 5) mild motor deficit, without ataxia or sensory loss. Exclusion criterion was previous history of any type of neuromuscular, musculoskeletal or severe cardiovascular disorders.

\subsection{Procedure}

Patients were randomised into 2 groups: the intervention group and the control group. The initial assessment of kinematic and kinetic gait parameters was performed using a 3-dimensional gait analysis system. Patients of the intervention group also underwent medical exams (clinical examination by a cardiologist, chest $x$-ray, electrocardiogram, and echocardiogram).

Then, patients in the control group were instructed to continue their usual daily activities, while those in the intervention group followed an 8-week exercise programme. At the end of this period, patients were re-evaluated using the gait analysis system.

\subsection{Assessment}

A motion analysis system consisting of 6 infrared cameras (Vicon MX 0306012) was used. Cameras were arranged in a circular configuration 3 meters above the ground and had a shooting speed of $100 \mathrm{~Hz}$. Images were digitised with Nexus 1.3106 and Polygon software packages, while kinematic data processing and analysis was carried out using MatLab software.

The motion analysis system was synchronised with an acquisition and analysis system of ground reaction forces using Vicon's Nexus 1.3106 software. The recording and analysis system of ground reaction forces consisted of 2 piezoelectric force plates (Kistler, type 9281B11 and 9281CA, respectively), 2 load amplifiers and an analogue-to-digital converter. Digital signal acquisition, processing and analysis was carried out using Nexus 1.3106 and Polygon software packages.

For the acquisition of temporal gait parameters, an electronic timing system was used, consisting of an electronic timer and 2 pairs of photocells (reflective markers). The reliability of the method in assessing the mechanical gait parameters in patients with stroke has been demonstrated [14].

During the procedure, 16 spherical reflective surface markers, $14 \mathrm{~cm}$ in diameter were attached bilaterally to specific anatomical landmarks of the patients' lower limbs and pelvis (heels, second metatarsal heads, lateral malleoli, lateral femoral condyles, lateral aspect of the thighs in line with the lateral femoral condyles and the greater trochanters, lateral aspect of the shanks in line with the lateral femoral condyles and the lateral malleoli, and anterior and posterior superior iliac spines). Patients were asked to walk barefoot at self-selected speed and 5 trials were recorded. A trial was accepted if each foot hit a different force 
plate.

Given that kinematic and kinetic gait parameters are related to gait speed [15], it was decided to maintain patients' gait speed constant between the initial and the final measurement. For this purpose, an electrically driven stick was used, moving steadily and at a speed equal to each patient's normal walking speed, which was calculated using six trial attempts before the initial assessment. Then, patients were instructed to move at a steady distance behind the stick.

Kinematic parameters studied were hip flexion-extension angle, hip abduction-adduction angle, hip internal-external rotation angle, knee flexion-extension angle and ankle dorsal-plantar flexion angle. Kinetic parameters studied were hip flexion-extension moment, hip abduction-adduction moment, hip internal-external rotation moment, knee flexion-extension moment and ankle dorsal-plantar flexion moment.

\subsection{Intervention}

Patients were divided into 2 groups depending on the level of gait recovery. Each group comprised 3 patients. The exercise programme was based on AHA/ASA guidelines, and included strength, endurance and flexibility training, as well as neuromuscular activities. It lasted 8 weeks, with 3 hourly sessions per week (Table 2) [16].

\subsection{Statistical Analysis}

Statistical analysis was performed using Statistical Package for Social Sciences 23 (SPSS Inc., Chicago, IL). We compared the initial and the final evaluation of each patient individually. Root mean squares (RMS) of each gait parameter were calculated for each $10 \%$ of the gait cycle. Differences between the RMS values between initial and final measurements were ascertained by repeated-measures Analysis of Variance (ANOVA), with one repeated factor, lower limb (paretic-non paretic) and time interval (initial and final measurement). Significance was defined as $5 \%$ (two-tailed $\mathrm{p}<0.05$ ).

Table 2. Content of exercise programme.

\begin{tabular}{ll}
\hline \multicolumn{1}{c}{ Exercise Type } & \multicolumn{1}{c}{ Intensity/Frequency/Duration } \\
\hline Aerobic ability & $55 \%-80 \%$ of maximal heart rate (11 - 14 RPE) \\
$\begin{array}{l}\text { Large-muscle activities (e.g., walking, stationary } \\
\text { cycle, functional activities from a seated position) }\end{array}$ & $\begin{array}{l}3 \text { days/week } \\
40 \text { minutes/session }\end{array}$ \\
$\underline{\text { Strength }}$ & $1-3$ set(s) of $10-15$ repetitions for $8-10$ \\
Resistance exercises with free weights, elastic & exercises involving the major muscle groups \\
bands, etc.-circuit training — functional mobility & 2 days/week \\
Flexibility & 2 days/week \\
Stretching (torso, upper and lower limbs) & Duration of each stretch: $10-30 \mathrm{sec}$. \\
Neuromuscular coordination & 2 days/week \\
Balance and coordination exercises & 20 minutes/session \\
\hline
\end{tabular}

RPE: Rated Perceived Exertion. 


\section{Results}

Table 3 and Table 4 show the distribution of patients according to any changes in the RMS values of each parameter. Most patients in the control group exhibited significant reductions in gait kinematics, and non-significant changes in gait kinetics in both lower limbs. Conversely, most patients in the intervention group showed significant increase or no change in joints' angles. As far as joint moments are concerned, most patients showed significant increases in all joints at the anterior-posterior plane, and non-significant changes in the frontal and transverse planes.

Figure 1 and Figure 2 show the mean waveforms of each gait parameter, both for the intervention and the control group, during the initial and final measurement. There was an obvious increase in the joints range of motion in the sagittal plane during the final measurement, both in the paretic and non-paretic lower limbs, throughout the movement path in the intervention group. Similarly, there was an increase in abduction and adduction hip movement as a result of the intervention. In the control group, however, there was a decrease in the range of motion of the above joints during the final measurement. As regards kinetic parameters, changes appear purely in the support phase, during the first $60 \%$ of the walking cycle.

Table 3. Comparative table of the change in kinematic and kinetic parameters of gait between the initial and final measurement-intervention group.

\begin{tabular}{ccccccc}
\hline \multirow{2}{*}{ INTERVENTION GROUP } & Increase & Decrease & No change & Increase & Decrease & No change \\
\cline { 2 - 7 } & \multicolumn{5}{c}{ Paretic leg } & \multicolumn{3}{c}{ Non paretic leg } \\
\hline Flex/ext hip angle & 2 & 1 & 3 & 2 & 1 & 3 \\
& $(33.33 \%)$ & $(16.67 \%)$ & $(50.00 \%)$ & $(33.33 \%)$ & $(16.67 \%)$ & $(50.00 \%)$ \\
Ab/add hip angle & 2 & 2 & 2 & 1 & 3 & 2 \\
& $(33.33 \%)$ & $(33.33 \%)$ & $(33.33 \%)$ & $(16.67 \%)$ & $(50.00 \%)$ & $(33.33 \%)$ \\
Int/ext rot hip angle & 1 & 4 & 1 & 2 & 2 & 2 \\
& $(16.67 \%)$ & $(66.67 \%)$ & $(16.67 \%)$ & $(33.33 \%)$ & $(33.33 \%)$ & $(33.33 \%)$ \\
Flex/ext knee angle & 3 & 1 & 2 & 4 & 0 & 2 \\
Ankle angle & $(50.00 \%)$ & $(16.67 \%)$ & $(33.33 \%)$ & $(66.67 \%)$ & $(00.00 \%)$ & $(33.33 \%)$ \\
& 2 & 1 & 3 & 3 & 1 & 2 \\
Flex/ext hip moment & $(33.33 \%)$ & $(16.67 \%)$ & $(50.00 \%)$ & $(50.00 \%)$ & $(16.67 \%)$ & $(33.33 \%)$ \\
Ab/add hip moment & 3 & 2 & 1 & 4 & 1 & 1 \\
Int/ext rot hip moment & $(50.00 \%)$ & $(33.33 \%)$ & $(16.67 \%)$ & $(66.67 \%)$ & $(16.67 \%)$ & $(16.67 \%)$ \\
Flex/ext knee moment & $(16.67 \%)$ & $(16.67 \%)$ & $(66.67 \%)$ & $(16.67 \%)$ & $(16.67 \%)$ & $(66.67 \%)$ \\
Ankle moment & $(50.00 \%)$ & $(16.67 \%)$ & $(33.33 \%)$ & $(50.00 \%)$ & $(16.67 \%)$ & $(33.33 \%)$ \\
& 3 & 1 & 2 & 3 & 1 & 2 \\
& $(50.00 \%)$ & $(16.67 \%)$ & $(33.33 \%)$ & $(50.00 \%)$ & $(16.67 \%)$ & $(33.33 \%)$ \\
\hline
\end{tabular}

Number of patients (\% percentage). 
Hip flexion - extension angle
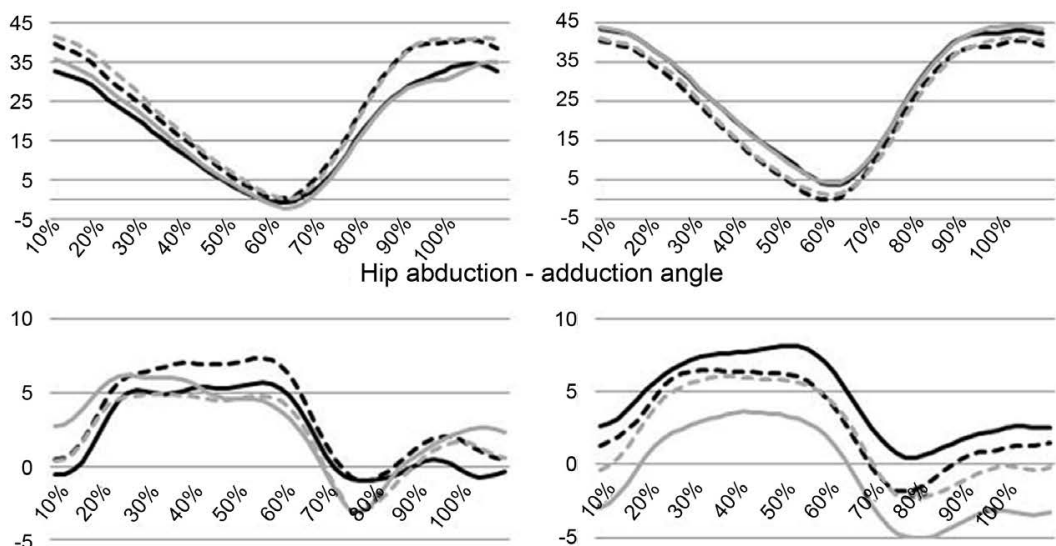

Hip internal - external rotation angle
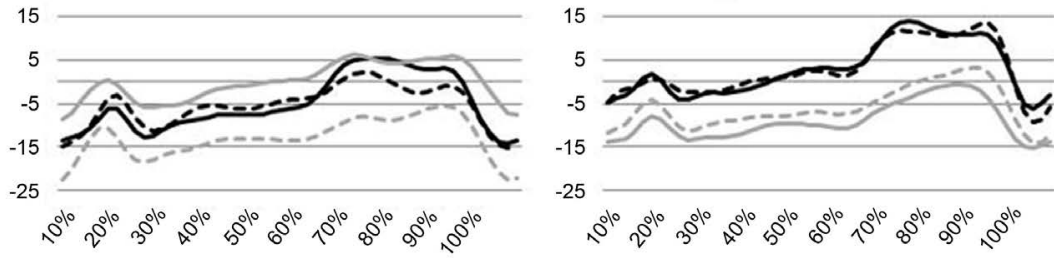

Knee flexion - extension angle
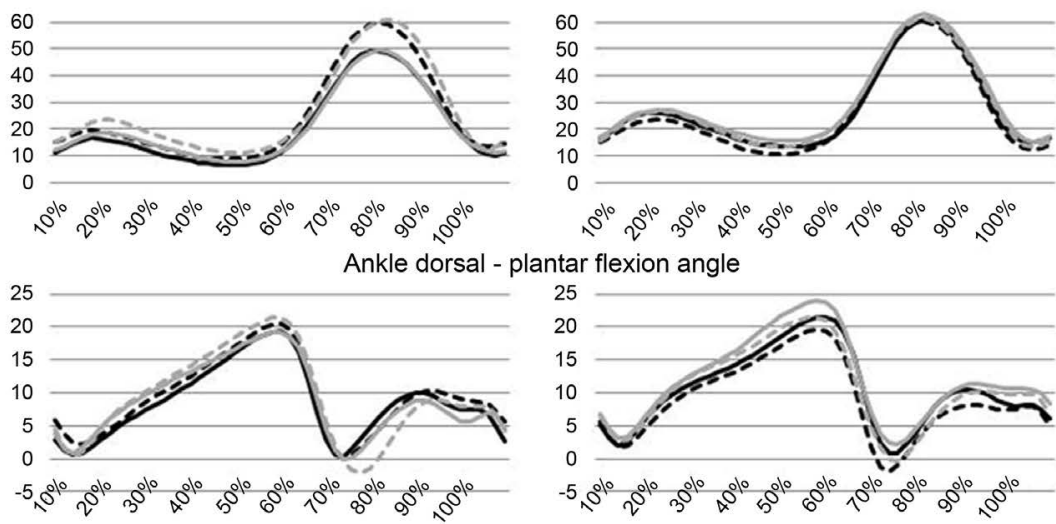

Figure 1. Waveforms of gait kinematics of paretic (black lines) and non-paretic (grey lines) lower limb during the initial (continuous lines) and the final (dashed lines) measurement. The left column presents the mean waveforms in the intervention group and the right column presents the waveforms in the control group.

Table 4. Comparative table of the change in kinematic and kinetic parameters of gait between the initial and final measurement-control group.

\begin{tabular}{ccccccc}
\hline \multirow{2}{*}{ CONTROL GROUP } & Increase & Decrease & No change & Increase & Decrease & No change \\
\cline { 2 - 7 } & \multicolumn{5}{c}{ Paretic leg } & \multicolumn{3}{c}{ Non paretic leg } \\
\hline Flex/ext hip angle & 2 & 3 & 1 & 2 & 3 & 1 \\
& $(33.33 \%)$ & $(50.00 \%)$ & $(16.67 \%)$ & $(33.33 \%)$ & $(50.00 \%)$ & $(16.67 \%)$ \\
Ab/add hip angle & 1 & 4 & 1 & 2 & 2 & 2 \\
& $(16.67 \%)$ & $(66.67 \%)$ & $(16.67 \%)$ & $(33.33 \%)$ & $(33.33 \%)$ & $(33.33 \%)$ \\
Int/ext rot hip angle & 1 & 3 & 2 & 1 & 3 & 2 \\
& $(16.67 \%)$ & $(50.00 \%)$ & $(33.33 \%)$ & $(16.67 \%)$ & $(50.00 \%)$ & $(33.33 \%)$ \\
Flex/ext knee angle & 1 & 3 & 2 & 1 & 2 & 3 \\
& $(16.67 \%)$ & $(50.00 \%)$ & $(33.33 \%)$ & $(16.67 \%)$ & $(33.33 \%)$ & $(50.00 \%)$ \\
\hline
\end{tabular}




\begin{tabular}{ccccccc} 
Continued & \multicolumn{7}{c}{} \\
\hline Ankle angle & 0 & 4 & 2 & 1 & 3 & 2 \\
& $(00.00 \%)$ & $(66.67 \%)$ & $(33.33 \%)$ & $(16.67 \%)$ & $(50.00 \%)$ & $(33.33 \%)$ \\
Flex/ext hip moment & 0 & 0 & 6 & 1 & 1 & 4 \\
& $(00.00 \%)$ & $(00.00 \%)$ & $(100.00 \%)$ & $(16.67 \%)$ & $(16.67 \%)$ & $(66.67 \%)$ \\
Ab/add hip moment & 1 & 1 & 4 & 1 & 0 & 5 \\
& $(16.67 \%)$ & $(33.33 \%)$ & $(66.67 \%)$ & $(16.67 \%)$ & $(00.00 \%)$ & $(83.33 \%)$ \\
Int/ext rot hip moment & 1 & 0 & 5 & 2 & 0 & 4 \\
Flex/ext knee moment & $(16.67 \%)$ & $(00.00 \%)$ & $(83.33 \%)$ & $(33.33 \%)$ & $(00.00 \%)$ & $(66.67 \%)$ \\
Ankle moment & $(16.67 \%)$ & $(16.67 \%)$ & $(66.67 \%)$ & $(16.67 \%)$ & $(00.00 \%)$ & $(83.33 \%)$ \\
& 1 & 1 & 4 & 1 & 0 & 5 \\
& $(16.67 \%)$ & $(16.67 \%)$ & $(66.67 \%)$ & $(16.67 \%)$ & $(00.00 \%)$ & $(83.33 \%)$ \\
\hline
\end{tabular}

Number of patients (\% percentage).
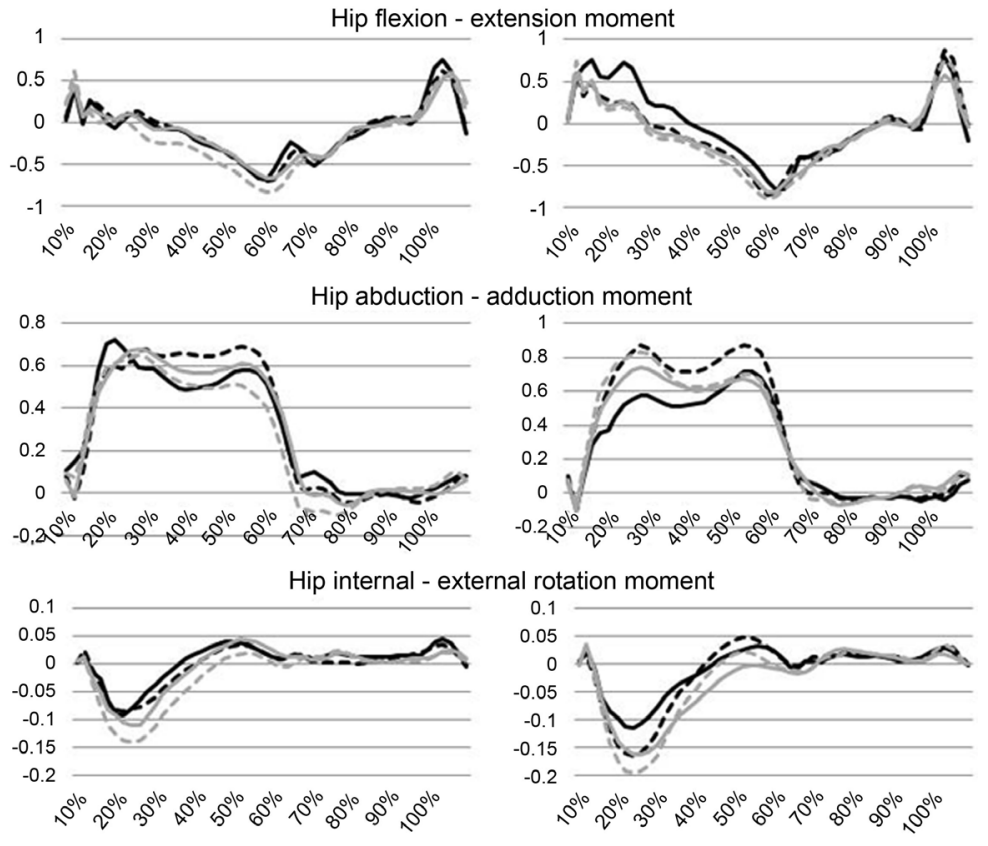

rnal rotation moment
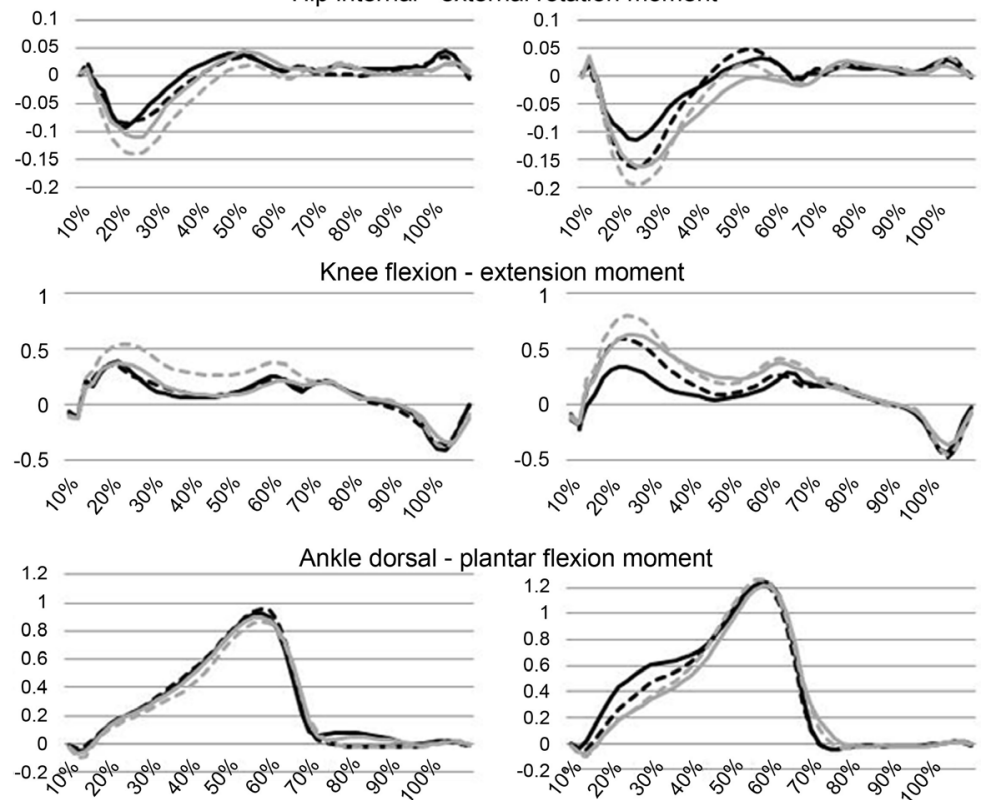

Figure 2. Waveforms of gait kinetics of paretic (black lines) and non-paretic (grey lines) lower limb during the initial (continuous lines) and the final (dashed lines) measurement. The left column presents the mean waveforms in the intervention group and the right column presents the waveforms in the control group. 


\section{Discussion}

The main findings of this study include significant reductions of gait kinematics in the control group, as opposed to significant increases or no changes, excluding hip movement at the transverse plane (internal-external rotation), in the intervention group.

Examining each parameter separately, increased hip flexion-extension angle after intervention is indicative of reduced spasticity of the extensor muscles and/or improvement of flexor muscle strength. Similarly, knee flexion increase in the swing phase is attributed to the improvement of the strength of the ankle plantar flexors and or hip flexors to achieve triple flexion in the lower limb (hipknee-ankle), as well as to the reduction of spasticity of the knee extensor muscles. An alternative or parallel explanation is the potential improvement of balance. Better balance eliminates the need to keep the lower limb close to the floor during the swing phase, to react promptly in case of loss of balance. On the other hand, the smaller extension angle during the support phase is indicative of improved strength and balance. Indeed, it is not required to search for a balance position through the locking of the knee in full extension.

Another interpretation of the above finding is the potential spasticity reduction of the gastrocnemius and the soleus muscles. Contrary to the intervention group patients, those in the control group showed greater knee extension during this phase in the final measurement, which demonstrates further worsening of the gait pattern as a result of time. The excessive extension of the knee during the final support prevents the normal flexion-extension pattern of the knee during the initial swing, and it does not allow effective forward movement. In this case, the person should either bring the limb through swinging to a more extended position or end up with a circular direction or hip elevation. However, each of the above manoeuvres is disadvantageous in terms of considering energy expenditure [17].

In the ankle joint, intervention group patients showed increased dorsiflexion during the final measurement. This finding points to increased strength of ankle dorsiflexors, and it also suggests the inhibition of the simultaneous plantar flexors' activation (reduction in spasticity). Furthermore, it may be an indication of greater balance, because patients did not choose an abnormal flat landing of the foot during initial foot contact to achieve increased base of support [17]. In contrast to patients in the intervention group, those in the control group exhibited more limited ankle dorsiflexion during the support phase, which indicates worsening of the gait pattern over time.

In kinetic parameters, most control patients had no significant changes, both in the paretic and the non-paretic lower limb. On the contrary, in the intervention group, there was a significant increase in mean moment values, both in the paretic and the non-paretic lower limb during movements at the anteroposterior plane in all three joints. This change reflects patients strengthening.

On average waveforms of moments, it was not possible to reflect changes 
made at individual level due to the different walking patterns of each patient. Each patient responded in his/her own special manner to the intervention programme. The different moment waveform patterns in stroke patients have been highlighted by Kim \& Eng [18]. In their study, moment waveforms that followed the normal pattern of healthy adults were recorded, as well as many variations. The different patterns of moments were correlated with gait speed. The same conclusion was drawn by Olney and Richards [17], who grouped these patterns with those based of patients' gait speed.

However, looking at the moment waveforms, it can be observed that for all joints in all the 3 planes in both groups, there were no significant changes between the initial and the final measurements in the swing phase, both in the paretic and non-paretic lower limb. However, small deviations were evident in the support phase. Therefore, the support phase was identified as the one that patients can utilise, through muscle strength increase, to alter their gait.

Our findings are concur with Teixeira-Salmela et al. In their study, the effect of a 10-week aerobic exercise and strengthening programme in patients with chronic stroke led to gait pattern improvement and an increase in selected kinematic and kinetic parameters [19].

The limitations include the small number of patients in each group, which in combination with the heterogeneous clinical feature of patients, did not allow group statistics to be performed. Therefore, to safely generalize the results it is advisable to consider a larger sample of patients. Another limitation was the lack of follow-up of patients beyond the two measurements, to record the extent to which the positive effect of exercise lasted over time. The final limitation relates to heterogeneity in the clinical features of stroke patients, which led to the absence of a common gait pattern to the whole patients. This, in combination to the small number of patients that met the inclusion criteria in the study, did not allow the use of group statistics and the direct comparison of patients in the intervention group with those in the control group.

\section{Conclusion}

In conclusion, gait kinematics was significantly increased in most patients in the intervention group. These changes were related with significant increases in gait kinetics during the support phase. It would now be useful to extend the observations to physical condition (spasticity, weakness, clinical characteristics of gait, etc.) in a larger patient sample.

\section{Conflicts of Interest}

The authors declare no conflicts of interest regarding the publication of this paper.

\section{References}

[1] Seshadri S., Beiser, A., Kelly-Hayes, M., Kase, C., Au, R., Kannel, W. and Wolf, P. 
(2006) The Lifetime Risk of Stroke. Estimates from the Framingham Study. Stroke, 37, 345-350. https://doi.org/10.1161/01.STR.0000199613.38911.b2

[2] Kwakkel, G., Kollen, B. and Lindeman, E. (2004) Understanding the Pattern of Functional Recovery after Stroke: Facts and Theories. Restorative Neurology and Neuroscience, 22, 281-299.

[3] Ashe, M., Miller, W., Eng, J. and Noreau, L. (2009) Older Adults, Chronic Disease and Leisure-Time Physical Activity. Gerontology, 55, 64-72. https://doi.org/10.1159/000141518

[4] Goljar, N., Burger, H., Vidmar, G., Leonardi, M. and Marincek, C. (2011) Measuring Patterns of Disability Using the International Classification of Functioning, Disability and Health in the Post-Acute Stroke Rehabilitation Setting. Journal of Rehabilitation Medicine, 43, 590-601. https://doi.org/10.2340/16501977-0832

[5] Rand, D., Eng, J., Tang, P., Jeng, J. and Hung, C. (2009) How Active Are People with Stroke? Use of Accelerometers to Assess Physical Activity. Stroke, 40, 163-168. https://doi.org/10.1161/STROKEAHA.108.523621

[6] Sullivan, K. and Cen, S. (2011) Model of Disablement and Recovery: Knowledge Translation in Rehabilitation Research and Practice. Physical Therapy, 91, 18921904. https://doi.org/10.2522/ptj.20110003

[7] Banks, G., Bernhardt, J., Churilov, L. and Cumming, T. (2012) Exercise Preferences Are Different after Stroke. Stroke Research and Treatment, 2012, Article ID: 890946. https://doi.org/10.1155/2012/890946

[8] Mead, G., Greig, C., Cunningham, I., Lewis, S., Dinan, S., Saunders, D., Fitzsimons, C. and Young, A. (2007) Stroke: A Randomized Trial of Exercise or Relaxation. Journal of the American Geriatrics Society, 55, 892-899. https://doi.org/10.1111/j.1532-5415.2007.01185.x

[9] Morris, J., Oliver, T., Kroll, T. and Macgillivray, S. (2012) The Importance of Psychological and Social Factors in Influencing the Uptake and Maintenance of Physical Activity after Stroke: A Structured Review of the Empirical Literature. Stroke Research and Treatment, 2012, Article ID: 195249.

https://doi.org/10.1155/2012/195249

[10] Rimmer, J., Wang, E. and Smith, D. (2008) Barriers Associated with Exercise and Community Access for Individuals with Stroke. Journal of Rehabilitation Research \& Development, 45, 315-322. https://doi.org/10.1682/JRRD.2007.02.0042

[11] Simpson, L., Eng, J. and Tawashy, A. (2011) Exercise Perceptions among People with Stroke: Barriers and Facilitators to Participation. International Journal of Therapy and Rehabilitation, 18, 520-530. https://doi.org/10.12968/ijtr.2011.18.9.520

[12] Resnick, B., Michael, K., Shaughnessy, M., Kopunek, S., Nahm, E. and Macko, R. (2008) Motivators for Treadmill Exercise after Stroke. Topics in Stroke Rehabilitation, 15, 494-502. https://doi.org/10.1310/tsr1505-494

[13] Billinger, S., Arena, R., Bernhardt, J., Eng, J., Franklin, B., Johnson, C., MacKay-Lyons, M., Macko, R., Mead, G. and Roth, E. (2014) Physical Activity and Exercise Recommendations for Stroke Survivors. Stroke, 45, 2532-2553. https://doi.org/10.1161/STR.0000000000000022

[14] Fotiadou, S., Aggeloussis, N., Gourgoulis, V., Malliou, P., Papanas, N., Giannakou, E., Iliopoulos, I., Vadikolias, K., Terzoudi, A. and Piperidou, H. (2018) Reproducibility of Gait Kinematics and Kinetics in Chronic Stroke Patients. Neurorehabilitation, 42, 53-61. https://doi.org/10.3233/NRE-172195

[15] Roth, E., Merbitz, C., Mroczek, K., Dugan, S. and Suh, W. (1997) Hemiplegic Gait. Relationships between Walking Speed and Other Temporal Parameters. American 
Journal of Physical Medicine \& Rehabilitation, 76, 128-133.

https://doi.org/10.1097/00002060-199703000-00008

[16] Thompson, W., Gordon, N. and Pescatello, L. (2009) American College of Sports Medicine-ACSM's Guidelines for Exercise Testing and Prescription. 8th Edition, LWW, Philadelphia, PA.

[17] Olney, S. and Richards, C. (1996) Hemiparetic Gait Following Stroke. Part I: Characteristics. Gait and Posture, 4, 136-148.

https://doi.org/10.1016/0966-6362(96)01063-6

[18] Kim, C. and Eng, J. (2004) Magnitude and Pattern of 3D Kinematic and Kinetic Gait Profiles in Persons with Stroke: Relationship to Walking Speed. Gait Posture, 20, 140-146. https://doi.org/10.1016/j.gaitpost.2003.07.002

[19] Teixeira-Salmela, L., Nadeau, S., Mcbride, I. and Olney, S. (2001) Effects of Muscle Strengthening and Physical Conditioning Training on Temporal, Kinematic and Kinetic Variables during Gait in Chronic Stroke Survivors. Journal of Rehabilitation Medicine, 33, 53-60. https://doi.org/10.1080/165019701750098867 